\title{
Inter- and intra-plant SC length variation in Crepis capillaris
}

\author{
D. DE AZKUE \& G. H. JONES \\ School of Biological Sciences, University of Birmingham, Birmingham B15 2TT, U.K.
}

\begin{abstract}
Synaptonemal complexes (SCs) were measured in large samples (16-31) of pachytene nuclei from five different plants of Crepis capillaris, with the aim of conducting a systematic study of intra-plant and interplant SC length variation. Individual plants showed considerable variation in SC length among cells (average range $=1.83 x$ ), which is presumed to reflect stage-related differences in chromosome and SC length during pachytene. The five plants selected for study showed highly significant differences in SC length. These differences are interpreted as real differences in the means and ranges of SC lengths in different genotypes rather than, for example, sampling effects. Mean SC lengths at pachytene and mean chiasma frequencies at metaphase I show signs of being positively related, but the correlation is statistically non-significant, indicating a need for more extensive study.
\end{abstract}

Keywords: Crepis capillaris, length, pachytene, synaptonemal complex, variation.

\section{Introduction}

Since its original discovery almost 40 years ago (Moses, 1956; Fawcett, 1956) the synaptonemal complex (SC) has been the subject of extensive investigation and characterization, initially at the ultrastructural level and, more recently, at the molecular level (Heyting et al., 1987). With the introduction of methods for analysing entire nuclear complements of SCs by three-dimensional reconstruction from serial sections and later by surface-spreading procedures, SC length measurements have become relatively routine and are widely reported in the literature (reviewed by Von Wettstein et al., 1984). SC length variation is of interest from a number of different perspectives. Despite the marked evolutionary conservation of SC ultrastructure, average SC length varies enormously across the phylogenetic range of eukaryotes, and there is considerable interest in relating this inter-specific variation to differences in genome size and genome composition with the aim of better understanding the quantitative and qualitative nature of the SC-genome interaction (e.g. Gillies, 1984; Anderson, et al., 1985).

Relatively less direct attention has been paid to the analysis of intra-specific SC length variation. Although $\mathrm{SC}$ length measurements are widely reported, most

Correspondence: Dr G. H. Jones, School of Biological Sciences, The University of Birmingham, Birmingham B15 2TT, U.K. accounts are relatively brief and superficial and, apart from a few studies, the extent and significance of SC length variation among and within individuals of particular species have not been systematically investigated. The majority of published accounts are uninformative in this respect because of low sample sizes (i.e. low numbers of nuclei analysed) or because the grouping of data from different individuals obscures any interindividual differences which might exist. In a few somewhat exceptional situations, however, some quite marked intraspecific SC length variations have been reported. These concern the effects of sex differences (e.g. Bojko, 1985; Wallace \& Hulten, 1985; Jones \& Croft, 1989) or supernumerary (B) chromosomes (Jones et al., 1989) which, in some cases at least, are associated with pronounced differences in average SC lengths and with substantial correlated differences in recombination frequencies.

The present study sets out to investigate and analyse SC length variation, unrelated to sex differences or B chromosomes, in normal diploid natural-population plants of the cytogenetically favourable species, Crepis capillaris. It is addressed particularly to characterizing the cell-to-cell variation in SC length which exists within individual plants, and also to investigating whether significant differences in average SC length occur among different plants. A supplementary aim is to investigate whether such inter-individual SC length variation (if present) is correlated with variation in 
chiasma frequency. The use and value of SC data for karyotyping will be discussed separately in a future paper.

\section{Materials and methods}

All five plants of Crepis capillaris used in this study were recently derived from natural population collections. Plants 1-4 were grown from achenes collected from a natural population on Bredon Hill, South Worcestershire, while Plant 5 was a second generation derivative of a natural population growing on the campus of the University of Birmingham. While, therefore, being fairly representative of natural population material, these plants cannot be claimed to be a random sample or cross-section of natural populations because they were deliberately chosen, on the basis of cytological screening, to give a wide range of mean chiasma frequencies.

The plants were overwintered as rosettes and flowered during the following spring.

\section{Surface-spreading of SCs}

The surface spreading procedure used was basically that described by Jones et al. (1989) with some modifications. Six to seven immature florets containing pollen mother cells judged to be in early prophase I of meiosis were gently tapped out in one drop of digestion medium $(0.1 \mathrm{~g}$ snail gut enzyme [cytohelicase], $0.250 \mathrm{~g}$ polyvinylpyrrolidone, and $0.38 \mathrm{~g}$ sucrose, in $25 \mathrm{ml}$ distilled water) in a cavity slide, and left for 3-4 min. Single small drops of this suspension were then transferred onto single drops of detergent solution ( 1 per cent Lipsol) on plastic-coated and glow-discharged glass slides. After 5-6 min, two drops of paraformaldehyde fixative were added, and the slides were then dried at $25-30^{\circ} \mathrm{C}$ on a hotplate for not less than six hours, rinsed in distilled water and air dried. For silver staining, a few drops of 50 per cent $\mathrm{AgNO}_{3}$ solution were placed onto slides, that were then covered with patches of nylon gauze and incubated in a moisture chamber at $60^{\circ} \mathrm{C}$ for $60 \mathrm{~min}$. Suitably spread nuclei were identified by bright field light microscopy and their positions on the slide marked with a fine pen. The plastic film was cut with a sharp blade and floated off the slides onto a clean water surface. Electron microscope grids were carefully placed over spread nuclei on the film, picked up from below onto steel meshes, and carefully dried. Suitably spread and stained nuclei were examined and photographed in a Philips EM301 or in a JEOL 1200EX electron microscope. Measurements of SCs were made from enlarged photographic prints (Fig. 1), using a Summagraphics digitizing tablet linked to a $\mathrm{BBC}$ microcomputer.

\section{Chiasma analysis}

Chiasma frequencies were scored from orcein stained squash preparations of 40 metaphase I pollen mother cells (p.m.c.) per plant.

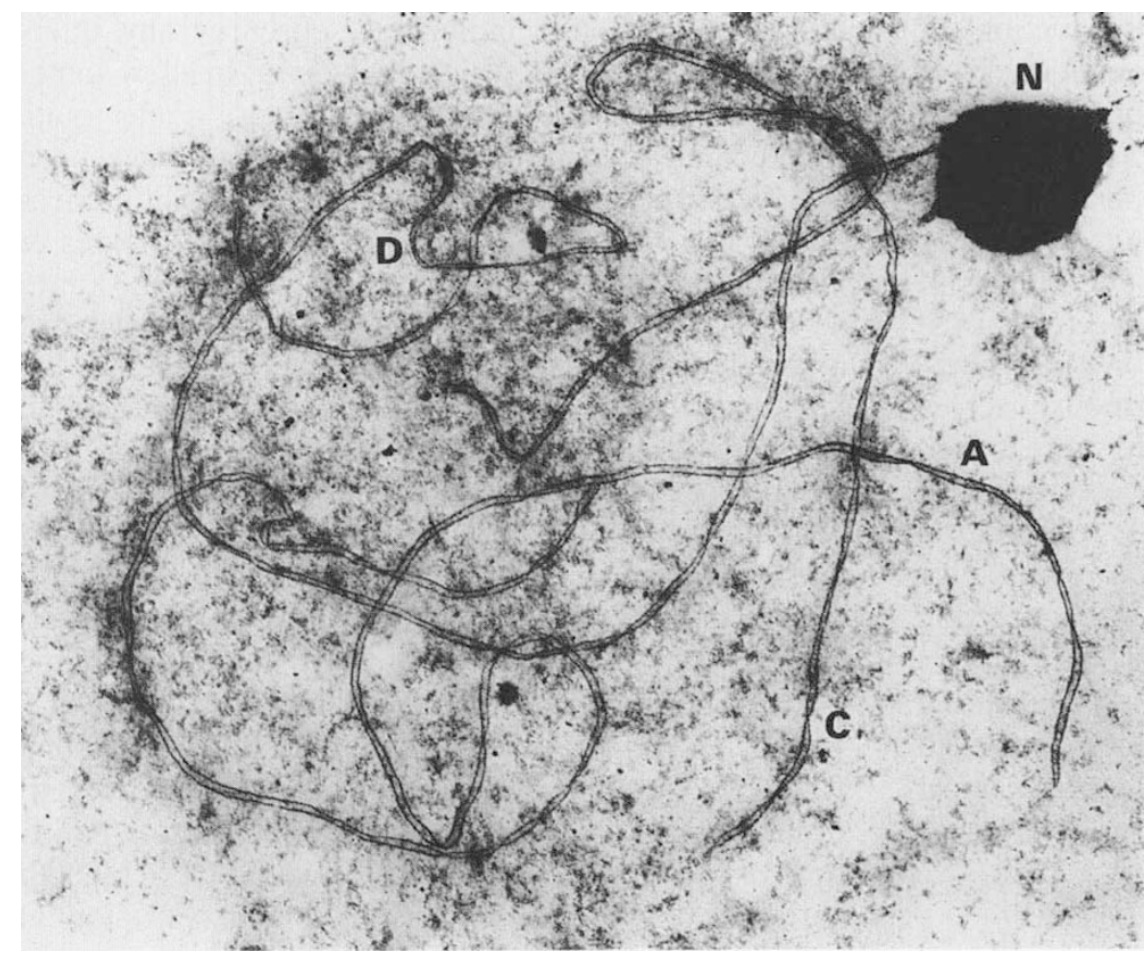

Fig. 1 Electron micrograph of a surface-spread and silver stained pachytene nucleus of Crepis capillaris showing the typical appearance of three SCs (labelled A,C,D). The D SC is associated terminally with a prominent nucleolus $(\mathrm{N})$. Magnification $=\times 6176$. 


\section{Results and discussion}

Total nuclear SC lengths were measured in as many pachytene nuclei (Fig. 1) as possible in each of the five plants selected for study. For the purposes of this study, and in order to maximize the numbers of nuclei per plant, total SC lengths were measured from some nuclei (13 in total) in which detailed identification of the three different SCs was not possible due to one or more regions of unresolvable tangles or overlaps. Nuclei in which SCs were obviously stretched or distorted were excluded. The total numbers of measured pachytenes ranged from 16 to 31 in different plants totalling 105 nuclei altogether (Table 1).

\section{Intra-plant variation in SC length}

All five plants show considerable ranges of total pachytene SC lengths, varying from $1.64 x$ in Plant 5 to $2.09 x$ in Plant 1 . The average range of within plant SC lengths over all five plants is $1.83 x$, whereas the total range of observed SC lengths over all five plants is $2.53 x$. These ranges are similar to those observed in a

Table 1 A summary of SC length data for five different plants of Crepis capillaris

\begin{tabular}{lllll}
\hline & $\begin{array}{l}\text { Mean SC } \\
\text { Plant }\end{array}$ & & & \\
& length & s.d. & $n$ & Range \\
\hline 1 & 140.95 & 20.48 & 22 & $100.87-210.74(2.09 \mathrm{x})$ \\
2 & 141.28 & 22.01 & 18 & $114.21-199.75(1.75 \mathrm{x})$ \\
3 & 169.89 & 18.60 & 31 & $130.57-223.16(1.71 \mathrm{x})$ \\
4 & 161.56 & 31.42 & 18 & $123.96-241.71(1.95 \mathrm{x})$ \\
5 & 203.28 & 23.57 & 16 & $154.94-254.85(1.64 \mathrm{x})$ \\
\hline
\end{tabular}

wide diversity of other organisms (Table 2) suggesting that they reflect some fundamental aspect of meiotic chromosome behaviour. However, relatively few direct comparisons with published intra-individual SC length variations are possible because of the common practice, in many studies, of pooling or grouping observations from different individuals. In some but apparently not all studies this appears to have been carried out to compensate for the low numbers of nuclei analysed per individual. In those few published cases where SC lengths are given separately for different individuals, similar ranges of SC lengths were observed to those found in individuals of Crepis capillaris (Holm \& Rasmussen, 1977; Croft, 1984; Gillies \& Cowan, 1985; de Jong et al., 1989).

Anderson et al. (1985) argued that the range of SC lengths observed in several Angiosperm plant species were mainly a reflection of stage-related differences in chromosome length during pachytene and considered that technical causes, such as physical stretching of SCs during preparation, were relatively unimportant. Since most workers introduce precautions to avoid including nuclei affected by technical artefacts of the spreading procedure this conclusion is probably generally applicable, but the possibility of some variation due to stretching cannot be excluded. Progressive variation in SC length during pachytene has been carefully analysed in sectioned Drosophila oocytes (Carpenter, 1979) making use of the developmental progression and ordering of cysts within germaria. This study recorded a $1.8 x$ range of SC lengths among pachytene oocytes and also concluded that SC lengths initially decreased during early to mid pachytene before extending again during late pachytene. A similar pattern of change in SC length was described in hamster spermatocytes, where sub-stages of pachytene

Table 2 A summary of published data on SC length variation. SS = surface spreading; $3 \mathrm{DR}=$ three-dimensional reconstruction; $n_{\mathrm{i}}=$ number of individuals; $n_{\mathrm{c}}=$ number of cells, presented as number of cells per individual where this is given

\begin{tabular}{|c|c|c|c|c|c|c|}
\hline Species & Method & $\begin{array}{l}\text { Mean SC } \\
\text { length }\end{array}$ & $n_{\mathrm{i}}$ & $n_{\mathrm{c}}$ & Range & Reference \\
\hline Homo sapiens & 3DR & 231 & 5 & $6,6,3,4,3$ & $1.30 x$ & Holm \& Rasmussen (1977) \\
\hline Homo sapiens & SS & 258.7 & 3 & 22 & $1.36 x$ & Solari $(1980)$ \\
\hline Cricetulus griseus & SS & 116.2 & 7 & 72 & $1.67 x$ & Moses et al. (1977) \\
\hline Felis catus & SS & 207.6 & 2 & 6,7 & $1.71 x$ & Gillies \& Cowan (1985) \\
\hline Lycopersicon esculentum & SS & 207.4 & $?$ & 100 & $1.94 x$ & Sherman \& Stack (1992) \\
\hline Triticum aestivum & SS & 1474 & $?$ & 20 & $1.65 x$ & $\operatorname{Holm}(1986)$ \\
\hline Secale cereale & SS & 664.2 & 4 & $3,5,5,15$ & $1.95 x$ & De Jong et al. (1989) \\
\hline Lilium longiflorum & SS & 3149 & 4 & $11,1,2,6$ & $1.93 x$ & Stack et al. (1989) \\
\hline Schizophyllum commune & 3DR & 23.2 & $?$ & 15 & $2.38 x$ & Carmi et al. (1978) \\
\hline
\end{tabular}


were defined by changes in XY morphology (Moses et al., 1977). Many plant species lack objective criteria for the reliable sub-staging of pachytene, but stagedependent alterations in SC length have nevertheless been described or inferred in some plants. In tomato, Stack \& Anderson (1986) relied on features of SC morphology, especially the appearance of the kinetochore to sub-stage pachytene. They found that the SCs underwent a progressive shortening throughout pachytene. In some other species where sub-staging is not possible the extent of pachytene chromosome contraction can be judged by comparison of very late zygotenes and very early diplotenes; in Zea mays for example an overall reduction in length of $2.8 x$ was deduced by this means (Gillies, 1983). The ranges of SC lengths observed in Crepis capillaris are similar to those described in maize which supports the contention that the pachytene nuclei analysed in the present study represent the entire range of pachytene.

The pattern of variability of SC lengths within the five plants of Crepis capillaris is clearly evident from the histograms in Fig. 2. While there are too few observations to be able to examine the statistical properties of these distributions (e.g. normality), it is nevertheless noticeable that all the plants display signs

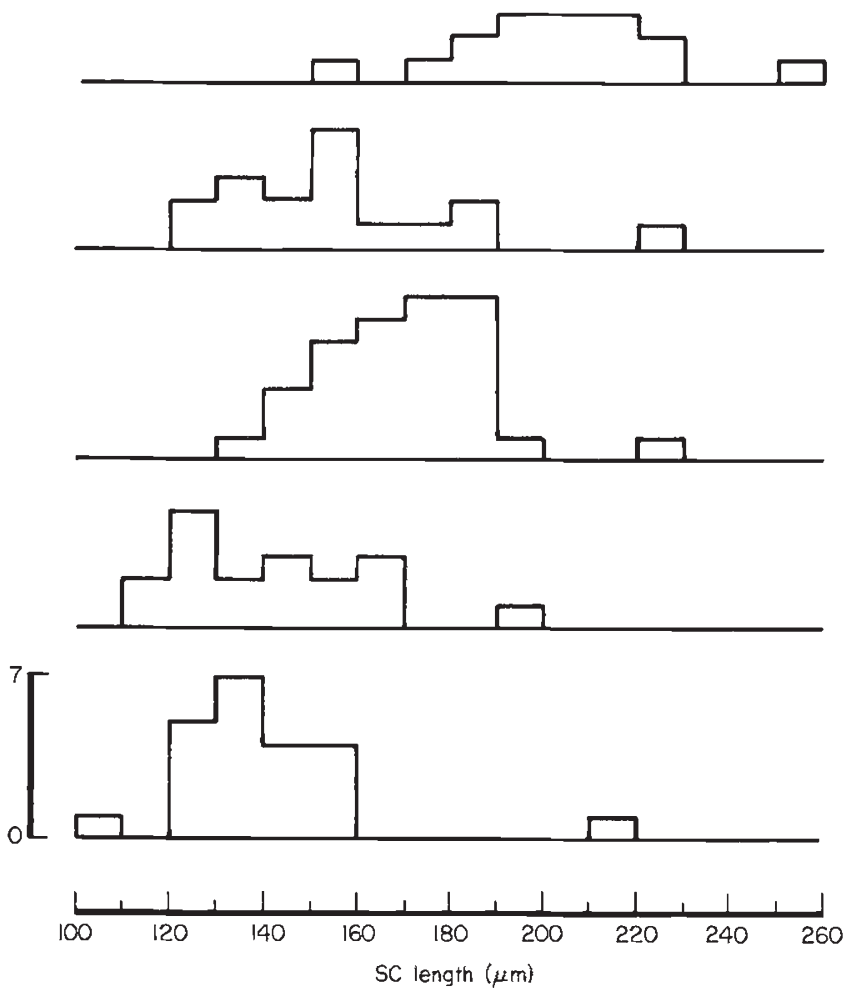

Fig. 2 Frequency histograms showing the range and distribution of nuclear SC lengths in each of five different plants of Crepis capillaris. of distinctly skewed distributions of SC length with at least one nucleus from each plant having exceptionally long SCs. It is interesting that maize SCs show a similarly skewed length distribution (Gillies, 1983). Moses et al. (1977) presented their data on SC lengths in 72 hamster pachytene nuclei in a similar way. They concluded that the distribution departed significantly from normality and showed evidence of multiple modes. However, since their histogram consisted of grouped data from no less than seven different individual animals these features of the distribution could well conceal significant inter-animal differences in SC length.

\section{Inter-plant SC length variation}

Figure 2 reveals that although the different plants show similar shaped distributions of SC length which overlap to a considerable extent, the mean and modal SC lengths of the different plants show quite substantial differences. This is confirmed by an analysis of variance, which shows that individual plants do in fact differ significantly in SC length $\left(\mathrm{VR}_{4,100}=22.194\right.$, $P<0.001)$. Since Plant 5 was derived from a different population than the other four plants, and has a markedly higher chiasma frequency, the analysis of variance was repeated excluding Plant 5 . This analysis shows that, even excluding Plant 5, the remaining four plants (all derived from the same population) also differ significantly in SC length $\left(\mathrm{VR}_{3,84}=7.304\right.$, $P<0.001)$. It was argued earlier that the nuclei sampled from individual plants probably represented the entire range of pachytene 'sub-stages'. This supports the interpretation that the plants genuinely differ in SC length and the differences detected are not simply the result of sampling different sub-stages of pachytene in different plants. The analysed nuclei, for individual plants, come from several (12-36) different florets prepared on between 2 and 6 different occasions, which strengthens this argument still further. This inter-plant variation is perhaps not surprising considering that Crepis capillaris is a genetically heterogeneous outbreeding species in the wild. Significant inter-strain, as opposed to inter-individual, differences in SC length have been reported previously in Zea mays (Gillies, 1983). Inter-individual SC length variation is rather infrequently reported or even considered in the literature. Significant differences between individuals are, however, reported from cats (Gillies \& Cowan, 1985) and also rye (de Jong et al., 1989) but the numbers of nuclei analysed per individual were relatively low in these studies (See Table 2).

This study give some notion of the variation in SC length within individual plants and among plants drawn 
from natural populations. Although the numbers of nuclei analysed from individual plants are high (16-31) compared to many previous studies, the extensive range of SC lengths within individual plants means that even higher numbers of nuclei are required to completely characterize the intra-plant SC length distribution. Because the five plants selected for study were not a random sample of population plants, the inter-plant variation in SC length is not representative. However, random samples of only five plants might show relatively little variation, hence necessitating much larger samples of plants and further escalating the scale of such a study.

\section{SC length variation in relation to chiasma frequency}

The plants included in this study showed a range of mean chiasma frequencies between 3.57 and 4.42 per cell. These values are representative of the range of mean cell chiasma frequencies observed in a large-scale natural population survey of this species (Whitehouse et al., 1981). Plotting these mean chiasma frequencies against mean pachytene SC lengths (Fig. 3) reveals evidence of a positive relationship of these variables. However, the correlation coefficient, though positive and large $(r=+0.781)$, is not significantly different from zero $\left(t_{(3)}=2.165 ; P>0.1\right)$, one explanation for which could be the relatively low number of plants in this study.

At present, and until more extensive studies can be carried out, there is only a tentative suggestion that mean SC length may be correlated with mean chiasma frequency in normal natural population plants of Crepis capillaris. This suggestion is, however, interesting and certainly merits further study as it is supported

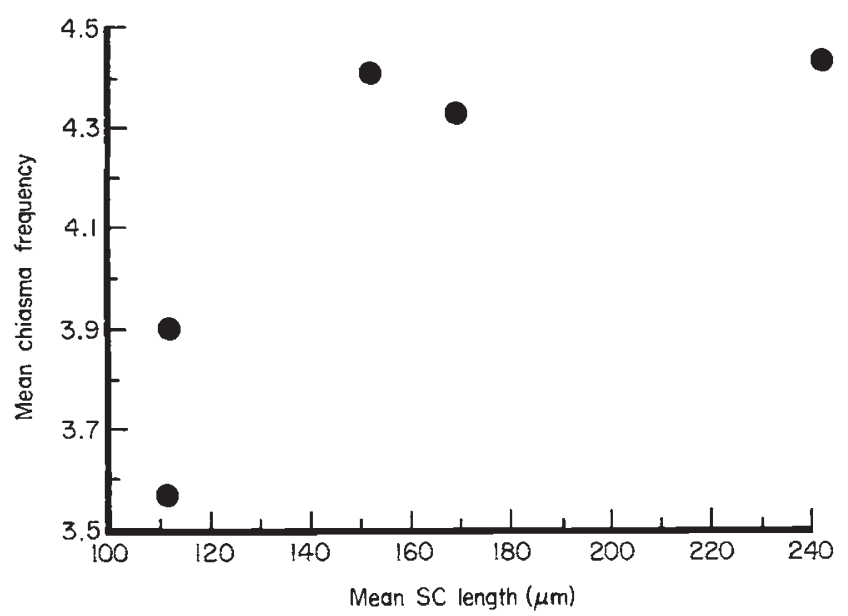

Fig. 3 Mean SC lengths of individual plants plotted against mean metaphase I chiasma frequencies. by indications from other studies for the existence of such a relationship. However, these other studies, as previously mentioned, involve special situations such as sex difference (e.g. Bojko, 1985; Wallace \& Hulten, 1985; Jones \& Croft, 1989) or possession of extra heterochromatin such as B chromosomes (Jones et al., 1989) or heterochromatic knobs (Mogensen, 1977). It would considerably strengthen the case for a direct involvement of the $\mathrm{SC}$ in regulating recombination if a correlation of SC length and chiasma frequency could be clearly demonstrated in normal natural population material, excluding the effects of sex difference or extra heterochromatin.

\section{References}

ANDERSEN, L. K., STACK, S. M., FOX, M. H. AND CHUANSANG, Z. 1985. The relationship between genome size and synaptonemal complex length in higher plants. Exp. Cell Res., 156, 367-378.

волко, м. 1985. Human meiosis IX. Crossing over and chiasma formation in oocytes. Carlsberg Res. Commun., 50, 43-72.

CARMI, P., HOLM, P. B., KOLTIN, Y., RASMUSSEN, S. W., SAGE, J. AND ZICKLER, D. 1978. The pachytene karyotype of Schizophyllum commune analysed by three-dimensional reconstruction of synaptonemal complexes. Carlsberg Res. Commun., 43, 117-132.

CARPENTER, A. T. C. 1979. Synaptonemal complex and recombination nodules in wild-type Drosophila melanogaster females. Genetics, 92, 511-541.

CROFT, J. A. 1984. Studies on chromosome synapsis and chiasma formation. Ph. D. Thesis, University of Birmingham. DE JONG, J. H., VAN EDEN, J. AND SYBENGA, J. 1989. Synaptonemal complex formation and metaphase I configuration patterns in a translocation heterozygote of rye (Secale cereale). Genome, 32, 72-81.

FAWCETT, D. W. 1956. The fine structure of chromosomes in the meiotic prophase of vertebrate spermatocytes. $J$. Biophys. Biochem. Cytol., 2, 403-406.

GILLIES, C. B. 1981. Electron microscopy of spread maize pachytene synaptonemal complexes. Chromosoma, 83, 575-591.

GILLIES, C. B. 1983. Ultrastructural studies on the association of homologous and non-homologous parts of chromosomes in the mid-prophase of meiosis in Zea mays. Maydica, 28, 265-287.

GILLIES, c. B. 1984. The synaptonemal complex in higher plants. CRC Crit. Revs Plant Sci., 2, 81-116.

GILLIES, C. B. AND COWAN, S. K. 1985. The pachytene synaptonemal complex complement of the cat. Genetica, 67, 99-107.

HEYTING, C. H., MOENS, P. B., VAN RAAMSDONK, W., DIETRICH, A. J. J., VINK, A. C. G. AND REDECKER, E. J. W. 1987. Identification of two major components of the lateral elements of synaptonemal complexes of the rat. Eur. J. Cell Biol., 43, $148-154$. 
HOLM, P. B. 1986. Chromosome pairing and chiasma formation in allohexaploid wheat, Triticum aestivum, analyzed by spreading of nuclei. Carlsberg Res. Commun., 51, 239-294.

HOLM, P. B. AND RASMUSSEN, S. w. 1977. Human meiosis I. The human pachytene karyotype analyzed by three-dimensional reconstruction of the synaptonemal complex. Carlsberg Res. Commun., 42, 283-323.

JONES, G. H. AND CROFT, J. A. 1989. Chromosome pairing and chiasma formation in spermatocytes and oocytes of Dendrocoelum lacteum (Turbellaria, Tricladida); a cytogenetical and ultrastructural study. Heredity, 63, 97-106.

JONES, G. H., WHITEHORN, J. A. F. AND ALBINI, S. M. 1989. Ultrastructure of meiotic pairing in B chromosomes of Crepis capillaris I. One-B and two-B pollen mother cells. Genome, 32, 611-621.

MOGENSEN, H. L. 1977. Ultrastructural analysis of female pachynema and the relationship between synaptonemal complex length and crossing-over in Zea mays. Carlsberg Res. Commun., 42, 475-497.

Moses, M. J. 1956. Chromosomal structures in crayfish spermatocytes. J. Biophys. Biochem. Cytol., 2, 215-218.

MOSES, M. J., SLATTON, G. H., GAMBLING, T. M. AND STARMER, C. F. 1977. Synaptonemal complex karyotyping in spermato- cytes of the Chinese hamster (Cricetulus griseus). III. Quantitative evaluation. Chromosoma, 60, 345-375.

SHERMAN, J. D. AND STACK, S. M. 1992. Two-dimensional spreads of synaptonemal complexes from solanaceous plants. V. Tomato (Lycopersicon esculentum) karyotype and idiogram. Genome, 35, 354-359.

SOLARI, A. J. 1980. Synaptonemal complexes and associated structures in microspread human spermatocytes. Chromosoma, 81, 315-337.

STACK, S. M. AND ANDERSON, L. K. 1986. Two-dimensional spreads of synaptonemal complexes from solanaceous plants II. Synapsis in Lycopersicon esculentum (tomato). Am. J. Bot., 73, 264-281.

STACK, S. M., ANDERSON, L. K. AND SHERMAN, J. D. 1989. Chiasmata and recombination nodules in Lilium longiflorum. Genome, 32, 486-498.

WALlACE, B. M. N. AND HULTEN, M. 1985. Meiotic chromosome pairing in the normal human female. Ann. Hum. Genet., 49, 215-226.

VON WETTSTEIN, D., RASMUSSEN, S. W. AND HOLM, P. B. 1984. The synaptonemal complex in genetic segregation. Ann. Rev. Genet., 18, 331-414.

WHITEHOUSE, C., EDGAR, L. A., JONES, G. H. AND PARKER, J. S. 1981. The population cytogenetics of Crepis capillaris I. Chiasma variation. Heredity, 47, 95-103. 